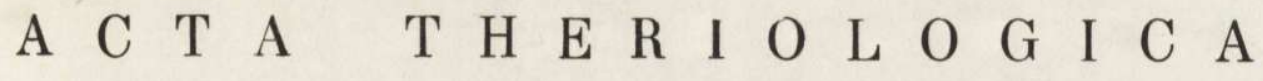 \\ VOL. XI, 8: 281-288.

\author{
Tadeusz ROSKOSZ, Franciszek KOBR Y N C Z K \\ und Henryk K O B R Y N
}

\section{Hydrometrische Bestimmung der Wirbelgrösse beim Rothirsch}

\author{
[Mit 3 Tabellen und 1 Abb.]
}

Die Autoren führten auf Wirbeln von 36 Hirschen $\left(27 \sigma^{7} \sigma^{7}\right.$ und 9 o $\circ$ ) Umfangsmessungen durch, wobei sie sich einer eigenen Methode bedienten, die sich auf das hydrometrische Gesetz von Archimedes stützt. Diese Methode zeichnet sich durch eine grosse Präzision aus, denn es wurden hier alle Kompenenten des Wirbels berücksichtigt, die doch vermittels Linienmassen sehr schwer zu erfassen sind. Sie ermöglicht ebenfalls das Ziehen von sehr eingehenden Folgerungen in Betreff sowohl der Grösse der einzelnen Wirbel wie auch der entsprechenden Abschnitte der Wirbelsäule. Die Autoren überprüften die postnatale Entwicklung der Pars praesacralis der Wirbelsäule und stellten die Mitabhängigkeit zwischen der Kapazität des Wirbelkanals und der Raummasse der sie umbauenden Knochenmasse fest.

\section{EINFUHRUNG}

Nach osteometrischen Methoden für die Wirbel suchend, fassten wir den Entschluss, den Versuch bei der Grössenbestimmung vermittels der Umfangmessung durchzuführen. Es scheint, dass diese Messung alle Komponenten des Wirbels umfasst, die doch bei der Linienmessung so schwer zu erfassen sind, aber gleichzeitig den Wert der ganzen Knochenmasse ausmacht, die das Wirbelloch umbaut.

Unsere Beobachtungen sind eine Kontinuation der Untersuchungen über die Wirbelsäule beim Rothirsch, Cervus elaphus Linnaeus, 1758 und sie haben als Vorgänger die Arbeit über das Thema der Kapazität des Wirbelkanals bei dieser Art (Roskosz \& Pytel, 1966).

\section{MATERIAL UND METHODE}

Das Untersuchungsmaterial bildeten Hals- Brust- und Lendenabschnitte der Wirbelsäulen von 36 Hirschen verschiedenen Alters und Geschlechts. Es wurde in vier Altersgruppen eingeteilt. Nähere Angaben in dieser Hinsicht enthält die oben zitierte Arbeit (Roskosz \& Py te 1, 1966).

Umfangmessungen auf einem unregelmässig gestelteten Körper, aber eben so einer ist ja der Wirbel, lassen sich nicht auf Grund von entsprechenden Linien- 
messungen durchführen. Es wurde eine Reihe von Methoden analysiert, mit Hilfe derer man diese Messung erreichen könnte. Eine von ihnen, die genaueste, wenn auch die älteste, ist die Mensurmethode, bei der die Raummasse des untersuchten Körpers mit der Kapazität der durch sie verdrängten Flüssigkeit gleich ist. Diese Methode ist die präziseste aber nur für kleine Objekte oder für solche mit angenähert dünner Walzenform, die sich in ein Gefäss mit schmalem Licht einführen lassen. Bei Mensuren mit grossem Durchschnitt, die man bei Wirbelumfangmessungen anwenden sollte, würden Niveauschwankungen der Flüssigkeit von 2-3 Millimeter Grösse auf der angefertigten Skala mit einer Genauigkeit bis zu $10 \mathrm{ml}$ unfassbar sein. Aus diesem Grunde versuchte man diese Methode auf solche Weise zu modifizieren, dass die nach dem Eintauchen des Wirbels hochgestiegene Flüssigkeitssäule vermittels eines kleinen Hahnes in ein daneben stehendes dünnes Mensurglas abgeführt wurde, das mit einer Genauigkeit bis zu $1 \mathrm{ml}$ messen konnte. Bei Anwendung dieser Methode gaben jedoch die sukzessiven Messungen eines Wirbels verschiedene Ergebnisse. Die Ursache der Ungenauigkeit lag in der sogar bei der kleinsten Erschütterung immer vibrierenden Flüssigkeitsoberfläche, die ihre eigene Oberflächenspannung besitzt, die im Moment der Beendigung der Messung verschiedene Niveaus auf der Höhe der Offnung verursacht. Der Hahn selbst wurde auch bis zu einem gewissen Grade zum Kapillargefäss, das osmotisch sogar dann die Flüssigkeit abzog, wenn ihr Niveau unterhalb der Öfnung stand. Aus diesem Grunde verzichtete man auf diese Methode. Es wurde ebenfalls versucht, die Raummasse des Körpers aus dem Quotient ihres Gewichtes und des spezifischen Gewichtes zu errechnen nach Formel:

$$
V=\frac{G}{G s}
$$

Aus Gründen des verschiedenen spezifischen Gewichtes der einzelnen Elemente des Knochengerüstes verwarf man auch diese Methode.

Die Autoren anerkannten die sich auf das hydrometrische Gesetz von Archimedes stützende Methode als die geeigneteste für Umfangmessungen der Wirbel. Bekanntlich gibt ein in denselben physischen Bedingungen und in zwei Milieus gewogener Körper zwei verschiedene Ergebnisse. Wenn man das Atmosphäre- und Wassermilieu annimmt, so wird der Unterschied dieser Ergebnisse in grosser Annäherung mit der Raummasse des untersuchten Körpers gleich sein, aber nur dann, wenn das spezifische Gewicht des Wassers mit der Einheit gleich ist.

Die Autoren benutzten zu Messungen Leitungswasser mit einer Temperatur von $+18^{\circ} \mathrm{C}$. Das Knochengewebe ist nach der Mazeration porös und seine Substantia spongiosa hat, besonders in den Ansätzen, eine Schwammstruktur. Um den Wirbel „massiv” zu machen, weichte man ihn vor der Durchführung der Messung im Wasser 90 Minuten lang und danach wog man ihn auf einer analytischen Waage mit einer Genauigkeit bis zu 1 g. Das zweite Ergebnis erhielt man, indem man den ins Wasser getauchten Wirbel wog, der an einen Draht angeheftet war, der wiederum an die Messchale befestigt worden war. Den Unterschied zwischen 
der ersten und zweiten Messung nahm man als Umfang des untersuchten Wirbels an auf Grund der Formel:

$$
V=a_{1}-a_{2}
$$

wo $V$ - die Raummasse, $a_{1}$ - die erste, $a_{2}$ - die zweite Messung bedeuten. Das sich im Gefäss unter der Waage befindende Wasser wurde für jede 10 Wirbel erneuert um die Bildung einer Lösung zu verhüten. Die Messungen eines jeden Wirbels wurden zweimal durchgeführt und danach errechnete man das arithmetische Mittel.

\section{ANALYSE DER ERGEBNISSE}

Der immer sieben Wirbel zählende Halsabschnitt weist eine grosse individuelle Variabilität auf, wenn es sich um die Raummasse der einzelnen Wirbel handelt und die Festsetzung irgendeiner Regelmässigkeit ist recht schwierig (Tab. 1, Abb. 1). Ganz unerwartet zeichnet sich der erste Wirbel, der optisch doch der grösste zu sein scheint, durch seine Umfangmessung überhaupt nicht aus. Das Vorhandensein der grössten Knochenmasse notierten wir im zweiten Wirbel, aber nur in der I, II und III Gruppe der Hirschbullen, obwohl in dieser letzten der sechste Wirbel mit dem Epistropheus der Grösse nach gleich ist. In der IV. Gruppe der Hirschbullen repräsentiert dieser Wirbel (der sechste) schon ganz selbständig die grösste Umfangmessung unter den übrigen Wirbeln des besprochenen Abschnittes. Bei Hirschkühen stellten wir die grösste Raummasse entweder im fünften (Gruppe I und III) oder im sechsten Wirbel (Gruppe II und IV) fest. Der an Raummasse bestimmt kleinste Wirbel im Halsabschnitt ist der siebente Wirbel sowohl bei Hirschbullen wie auch in einigen Gruppen bei Hirschkühen.

Sich in eine eingehende Analyse über den Grössenanwuchs der einzelnen Wirbel nicht einlassend, kann man feststellen, dass der Anwuchs der Knochenmasse der Wirbel des Halsabschnittes bei Hirschbullen mit verschiedener Intensität so lange andauert, bis er denjenigen Zustand erreicht, der in der Gruppe III auftritt, aber danach unterliegt er bei den ältesten Tieren einer Hemmung (Tab. 2). Bei Hirschkühen notiert man dagegen in den ersten drei Altersgruppen eine gewisse Stabilität im Anwuchs der Raummasse der Wirbel ja sogar ein geringes Absinken; in Gruppe IV aber ist sukzessiv ein ziemlich grosser Anwuchs sichtbar (um 20,3\% ) - vergl. Tab. 2 und Abb. 1.

Der Brustabschnitt wird am häufigsten durch 13 Wirbel repräsentiert, aber im Gegensatz zum Halsabschnitt zeichnet er sich durch eine grosse Regelmässigkeit aus, wenn es sich um die Umfangsmessungen der einzelnen Wirbel handelt (Tab. 1, Abb. 1). Die bestimmt grösste Knochenmasse besitzt also der erste Wirbel, von dem ab eine stufenweise Verrin- 
gerung der Umfangsmessungen der Wirbel beginnt, die sich am meisten bis zum zehnten Wirbel lang zieht. Diese Feststellung betrifft Individuen beiderlei Geschlechts ohne Rücksicht auf die Altersgruppen. In den weiteren Wirbeln wird abermals ein geringer Anwuchs der Messung notiert.

Der Grössenanwuchs der Konchenmasse der Wirbel im Brustabschnitt als Ganzes, ist mit dem Alter verbunden und kennzeichnet sich bei Hirschbullen durch einem dauernden Anwuchs beginnend von Gruppe I

Tabelle 1.

Umfangsmessungen im Mittel der Wirbel (in $\mathrm{cm}^{3}$ ) in den entsprechenden Altersgruppen.

\begin{tabular}{|c|c|c|c|c|c|c|c|c|c|}
\hline \multirow{2}{*}{\multicolumn{2}{|c|}{ Gruppe }} & \multicolumn{4}{|c|}{ H1rsahböcke } & \multicolumn{4}{|c|}{ H1rschkühe } \\
\hline & & I & II & III & IV & I & II & III & IV \\
\hline \multirow{8}{*}{$\begin{array}{l}\text { Hals- } \\
\text { abschn1tt }\end{array}$} & W1rbel & & & & & & & & \\
\hline & I & 83 & 112 & 144 & 137 & 68 & 56 & 63 & 64 \\
\hline & II & 93 & 134 & 163 & 141 & 75 & 64 & 67 & 78 \\
\hline & III & 71 & 120 & 144 & 142 & 63 & 68 & 63 & 74 \\
\hline & IV & 74 & 128 & 159 & 155 & 75 & 74 & 68 & 85 \\
\hline & v & 79 & 126 & 151 & 153 & 78 & 76 & 76 & 9. \\
\hline & VI & 78 & 116 & 163 & 159 & 77 & 82 & 73 & 103 \\
\hline & VII & 58. & 93 & 118 & 112 & 56 & 61 & 60 & 75 \\
\hline \multirow{13}{*}{$\begin{array}{l}\text { Brust- } \\
\text { absobn1t t }\end{array}$} & I & 54 & 86 & 105 & 102 & 50 & 56 & 58 & 67 \\
\hline & II & 41 & 77 & 94 & 93 & 42 & 47 & 47 & 61 \\
\hline & III & 39 & 70 & 86 & 88 & 40 & 43 & 41 & 54 \\
\hline & IV & 37 & 67 & 80 & 82 & 37 & 40 & 38 & 48 \\
\hline & v & 36 & 61 & 77 & 77 & 35 & 37 & 35 & 46 \\
\hline & VI & 33 & 54 & 68 & 69 & 30 & 35 & 34 & 42 \\
\hline & VII & 29 & 47 & 59 & 59 & 27 & 31 & 28 & 37 \\
\hline & VIII & 28 & 39 & 50 & 49 & 25 & 27 & 25 & 33 \\
\hline & IX & 27 & 36 & 44 & 45 & 23 & 25 & 22 & 30 \\
\hline & $x$ & 27 & 35 & 42 & 43 & 24 & 24 & 23 & 29 \\
\hline & XI & 30 & 35 & 42 & 44 & 25 & 24 & 24 & 30 \\
\hline & XII & 34 & 40 & 49 & 50 & 29 & 26 & 28 & 34 \\
\hline & $X I I I$ & 38 & 45 & 55 & 57 & 34 & 31 & 32 & 39 \\
\hline \multirow{6}{*}{$\begin{array}{l}\text { Lenden- } \\
\text { abschndtt }\end{array}$} & I & 41 & 47 & 67 & 69 & 41 & 42 & 38 & 46 \\
\hline & II & 46 & 62 & 77 & 79 & 46 & 39 & 44 & 54 \\
\hline & III & 51 & 65 & 82 & 84 & 49 & 48 & 48 & 59 \\
\hline & IV & 53 & 66 & 80 & 91 & 51 & 49 & 52 & 60 \\
\hline & $v$ & 53 & 71 & 84 & 87 & 54 & 52 & 52 & 6) \\
\hline & VI & 54 & 74 & 88 & 89 & 51 & 54 & 52 & 65 \\
\hline
\end{tabular}

bis IV, obwohl er in der letzten sehr gering ist (um 1,6\%). Bei Hirschkühen dagegen beobachtet man einen ungrossen Anwuchs in Gruppe II, aber dann ein Absinken in Gruppe III und nachfolgend abermals einen grossen Anwuchs in Gruppe IV (um 33,3\%) - Tab. 2.

Der aus sechs Wirbeln bestehende Lendenabschnitt weist ebenfalls bei den Umfangsmessungen der einzelnen Wirbel eine ziemlich grosse Beständigkeit auf, jedoch nicht eine so grosse wie im Brustabschnitt. Der 
Anwuchs der Knochenmasse vom ersten bis zum letzten Lendenwirbel ist als ob eine weitere Kontinuation des stufenweisen Anwuchses der Raummasse der letzten Brustwirbel (Tab. 1, Abb. 1). In einigen Altersgruppen (IV. der Hirschbullen und I. der Hirschkühe) ist diese Rechtmässigkeit erschüttert und der an Raummasse grösste Wirbel ist entwe-

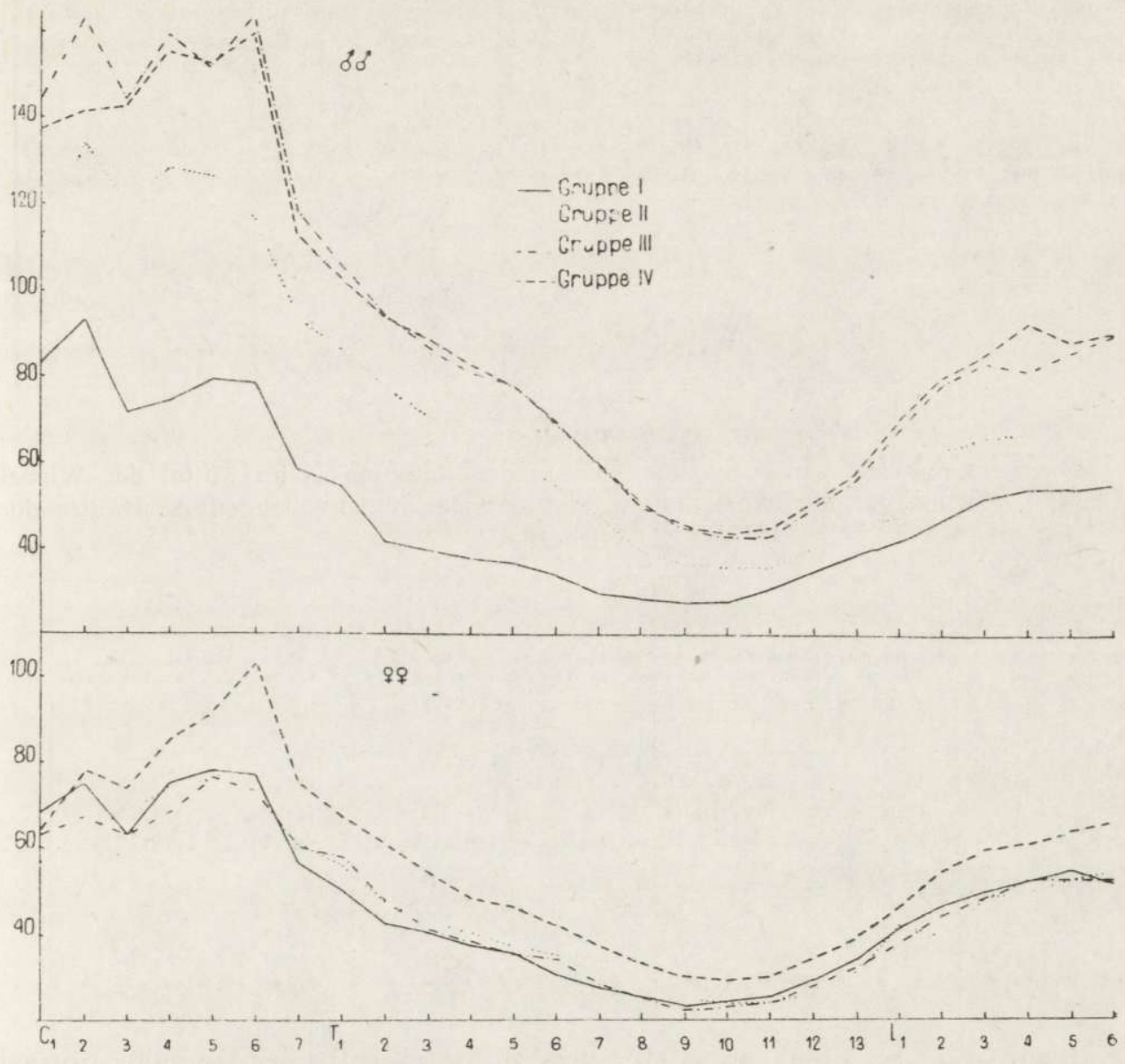

Abb. 1. Raummasse im Mittel der einzelnen Wirbel in Altersgruppen.

der der vierte oder der fünfte Wirbel, obwohl diese im Vergleich mit dem letzten Wirbel der entsprechenden Gruppen nur ein wenig grösser sind.

Die Summe der Raummasse der einzelnen Wirbel des Lendenabschnittes ist bei Hirschbullen in Gruppe I (Tab. 2) am kleinsten. Der Raummassenanwuchs der Knochenmasse des ganzen Abschnittes erstreckt sich bei diesem Geschlecht im verschiedenen Grade bis zur Gruppe IV; bei Hirschkühen dagegen ist die Raummasse in den ersten drei Gruppen fast 
Tabelle 2.

Summe der Raummasse im Mittel der einzelnen Wirbel für die entsprechenden Abschnitte der Wirbelsäule und ihr prozentsatzmässiger Anwuchs in den Altersgruppen.

\begin{tabular}{|c|c|c|c|c|c|c|c|c|}
\hline \multirow[b]{2}{*}{ Gruppe } & \multicolumn{2}{|c|}{ Ila1sabschntt } & \multicolumn{2}{|c|}{ Brustabschnitt } & \multicolumn{2}{|c|}{ Lendenabschnit } & \multicolumn{2}{|c|}{ Zusammen } \\
\hline & $\begin{array}{c}\text { Raurnasse } \\
\text { in } \mathrm{cm}^{3}\end{array}$ & $\begin{array}{c}\text { Anwuchs } \\
\text { in } x\end{array}$ & $\begin{array}{c}\text { Raunnasse } \\
1 \mathrm{n} \mathrm{cm}\end{array}$ & $\begin{array}{c}\text { Anwuchs } \\
\text { in } \%\end{array}$ & $\begin{array}{c}\text { Raunnasse } \\
1 \mathrm{n} \mathrm{cm}^{3}\end{array}$ & $\begin{array}{c}\text { Anwuchs } \\
\text { in } x\end{array}$ & $\begin{array}{c}\text { Rauminasse } \\
11 \mathrm{om}^{3}\end{array}$ & $\begin{array}{c}\text { Anwuchs } \\
\text { 1n } \%\end{array}$ \\
\hline & Harschbocke & & & & & & & \\
\hline 1 & 530 & & 453 & & 298 & & 1287 & \\
\hline II & 829 & $.54,7$ & 692 & $+52,7$ & 385 & $+29,2$ & 1906 & $+55,9$ \\
\hline 111 & 1042 & $+39,7$ & 851 & $+35,1$ & 478 & $+31,2$ & 2371 & $+28,3$ \\
\hline IV & 999 & $-8,0$ & 858 & $+1,6$ & 499 & $+12,7$ & 2356 & $-1,1$ \\
\hline & Hirschisute & & & & & & & \\
\hline 1 & 492 & & 421 & & 292 & & 1205 & \\
\hline II & 481 & $-2,2$ & 455 & $+5,9$ & 284 & $-2,8$ & 1211 & $+0,5$ \\
\hline III & 470 & $-2,3$ & 435 & $-2,6$ & 289 & $.0,7$ & 1191 & $-0,7$ \\
\hline IV & 570 & $+20,3$ & 350 & $+33,3$ & 347 & $+20,9$ & 1467 & $+22,9$ \\
\hline
\end{tabular}

Tabelle 3.

Prozentsatzmässiger Anwuchs der Summe der Raummasse im Mittel der Wirbel und der Kapazität der Wirbelkanäle der einander entsprechenden Abschnitte der Wirbelsäule.

\begin{tabular}{|c|c|c|c|c|c|c|}
\hline \multirow{2}{*}{ Gruppe } & \multicolumn{2}{|c|}{ Halsabschnitt } & \multicolumn{2}{|c|}{ Brustabschnitt } & \multicolumn{2}{|c|}{ Lendenabschnit $t$} \\
\hline & $\begin{array}{l}\text { Raumnasse } \\
\text { d. Wirbel }\end{array}$ & $\begin{array}{l}\text { Kapazıt täten } \\
\text { d. W1rbelkanals }\end{array}$ & $\begin{array}{l}\text { Raummasse } \\
\text { d. } \| 1 \text { rbe } 1\end{array}$ & $\begin{array}{l}\text { Kapazitäten } \\
\text { d. \#1rbelkanals }\end{array}$ & $\begin{array}{l}\text { Raummasse } \\
\text { d. N1rbel }\end{array}$ & $\begin{array}{l}\text { Kapazi tä te } \\
\text { d. ,yrbelkanals }\end{array}$ \\
\hline I & H1rschbö & cke & - & - & - & - \\
\hline II & $+54,7$ & $+47,5$ & $+52,7$ & $+42,0$ & $+29,2$ & $+36,5$ \\
\hline III & $+39,7$ & $+12,8$ & $+35,1$ & $+17,2$ & $+31,2$ & $+13,3$ \\
\hline IV & $-8,0$ & $-11,1$ & $+1,6$ & $-10,1$ & $+12,7$ & $-5,6$ \\
\hline I & Hirsohkü & be & - & - & - & - \\
\hline II & $-2,2$ & $+13,6$ & $+5,9$ & $+8,3$ & $-2,8$ & $+5,0$ \\
\hline III & $-2,3$ & $+4,6$ & $-2,6$ & $+5,8$ & $+0,7$ & $+2,6$ \\
\hline IV & $+20,3$ & $+19,2$ & $+33,3$ & $+17,5$ & $+20,9$ & $+7,3$ \\
\hline
\end{tabular}

gleich und erst ab Gruppe IV wird ihr beträchtlicher Anwuchs notiert (um 20,9\%) - Tab. 2.

Den Umfanganwuchs der Knochenmasse der Wirbel von Pars praesacralis der Wirbelsäule, als Ganzes, analysierend, kann man feststellen, dass ihr Anwuchs bei Hirschbullen in den Gruppe II und III beträchtlich vor sich geht, aber in Gruppe IV nur ein geringes Absinken eintritt. Bei Hirschkühen erhält sich diese Raummasse grundsätzlich in den ersten drei Altersgruppen auf demselben Niveau, aber in Gruppe IV kann man ihren beträchtlichen Anwuchs beobachten (um 22,9\%) - Tab. 2.

Interessant sieht die Zusammenstellung der Mitabhängigkeit des pro- 
zentsatzmässigen Anwuchses der Kapazität der Wirbelkanäle mit der Raummasse der sie umbauenden Knochenmasse der entsprechenden Abschnitte der Wirbelsäule aus (Tab. 3). Bei Hirschbullen kann man den Anwuchs beider Messungen in Gruppe II und III in allen besprochenen Abschnitten der Wirbelsäule beobachten. Wenn auch in der Gruppe der ältesten Tiere (IV) im Halsabschnitt nur eine ungrosse Verringerung beider Werte notiert wurde, so wurde in den Brust- und Lendenabschnitten die Veringerung der Kapazität der Wirbelkanäle dieser Abschnitte von einem ungrossen Anwuchs der Rummasse ihrer Knochenmasse begleitet.

Bei Hirschkühen sieht diese Situation ganz anders aus. Die Kapazität der Wirbelkanäle aller besprochenen Abschnitte wächst mit verschiedener Intensität von Gruppe II bis IV, aber die Raummasse des Knochenumbaues dieser Kanäle erhält sich in den Gruppen II und III ungefähr auf dem Niveau von Gruppe I und erst in der Gruppe IV beobachtet man ihren beträchtlichen Anwuchs.

\section{FOLGERUNGEN}

Die hydrometrische Methode, vermittels derer man die Raummasse der Knochen bestimmen kann, scheint ein gutes und unkompliziertes Mittel für osteometrische Beobachtungen zu sein. Die Berechnung nur einer Messung - der Raummasse - ersetzt eine ganze Reihe von Linienmessungen und Indices, die sogar bei der präzisesten Berechnung kein wirkliches Bild von der Grösse des Knochenobjektes geben.

Sich dieser Methode bedienend kann man ebenfalls eingehende Folgerungen ziehen, wenn es sich um den postnatalen Anwuchs des Knochengewebes sowohl der einzelnen Knochen wie auch der ganzen Abschnitte handelt, wobei hier alle Knochenvorsprünge berücksichtigt werden, die doch mit Hilfe von Linienmessungen so schwer durchzuführen sind.

Die Auswahl der Wirbel als Objekte zur Durchführung von hydrometrischen Versuchen war nicht zufällig. Sie stellen nämlich diejenigen Fragmente des Knochengerüstes dar, deren gänzliche Grösse mit Hilfe von Messungen ihrer anatomischen Einzelheiten am schwersten zu bestimmen ist. Dies bedeutet jedoch nicht, dass eine Umfangsmessung mit Erfolg bei einer Gelegenheit von Erwägungen über andere Teile des Kno= chengerüstes nicht angewandt werden könnte.

Obwohl das verhältnismässig spärliche Untersuchungsmaterial, besonders aber wenn es sich um Hirschkühe handelt, uns dazu zwang beim Ziehen von Folgerungen einen grossen Kritizismus zu bewahren, so muss dennoch unterstrichen werden, dass dank der Stützung der Untersuchungen auf Umfangsmessungen man eine ganze Reihe von interessanten Beobachtungen hat machen können. Die Verringerung der Kapazität des 
Wirbelkanals des Halsabschnittes in der Gruppe der ältesten Hirschbullen wird durch die Verringerung der Raummasse der Wirbel dieses Abschnittes begleitet. In den Brust- und Lendenabschnitten unterliegt die Kapazität der Wirbelkanäle mit dem Alter ebenfalls einer Verringerung, aber der Umfang der sie umbauenden Knochenmasse dagegen vergrössert sich ein wenig (Tab. 3).

Bei Hirschkühen steht der Anwuchs der Kapazität des Wirbelkanals aller untersuchten Abschnitte, der mit verschiedener Intensität von der füngsten Tiergruppe bis zur ältesten verläuft, nicht im Einklang mit dem Anwuchs der Wirbelraummasse dieser Abschnitte in den Gruppen II und III (Tab. 3). Die Aufrechterhaltung der Raummasse in diesen Gruppen auf dem Niveau von Gruppe I (der jüngsten Individuen) zeugt von einer eventuellen Erschütterungsmöglichkeit im Haushalt mit Kalk und Phosphor, die mit dem Zeugungsprozess verbunden ist.

Diese Mitteilung prätendiert nicht auf eine gänzliche Erschliessung dieses Problemes; nicht solche Absichten hatten schliesslich die Autoren. Es handelte sich hauptsächlich darum, um die Möglichkeit der Anwendung einer Methode für Bestimmung der Raummasse der Knochen erweisen zu können, die für osteometrische Untersuchungen eine schätzbare Ergänzung der bisher angewandten sein kann.

\section{SCHRIFTTUM}

1. Roskosz T. \& Pytel S., 1966: Ein Versuch zur Bestimmung des Wirbelkanals beim Rothirsch. Acta theriol., 11, 7: 269-280.

Received July 19, 1965.

Institut für Anatomie der Tiere

an der Landwirtschaftliche Hochschule,

Warszawa, ul. Grochowska 272.

Tadeusz ROSKOSZ, Franciszek KOBRYŃCZUK i Henryk KOBRYŃ

HYDROMETRYCZNE OKREŚLANIE WIELKOSCI KRĘGOW U JELENIA

Streszczenie

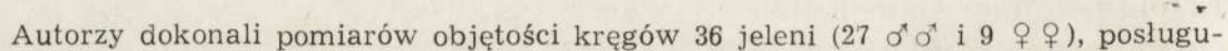
jąc się metodą własną opartą na hydrometrycznym prawie Archimedesa. Metoda ta ođznacza się dużą precyzyjnością, ponieważ brane są tu pod uwagę wszystkie komponenty kręgu tak trudne do uchwycenia pomiarami liniowymi. Pozwala ona również na wyciąganie bardziej szczegółowych wniosków dotyczących zarówno wielkości poszczególnych kręgów, jak i odpowiednich odcinków kręgosłupa. Autorzy prześledzili rozwój pozapłodowy odcinka przedkrzyżowego kręgosłupa oraz ustalili współzależność pomiędzy pojemnością kanału kręgowego a objętością obudowującej go masy kostnej. 